\title{
A Rare Complication of Dengue Fever
}

\author{
Waseem Dar' ${ }^{\star}$, Pervez Sofi', Reyaz Ahmad ${ }^{1}$, Gagan Chauhan1, Salil Singh² and Dakesh Dua ${ }^{3}$ \\ ${ }^{1}$ Department of Internal and Critical Care Medicine, Sanjeev Bansal Cygnus Hospital, Railway Road Karnal, Haryana, India \\ ${ }^{2}$ Department of Radiodiagnosis, Sanjeev Bansal Cygnus Hospital, Railway Road Karnal, Haryana, India \\ ${ }^{3}$ Department of Neurosurgery, Sanjeev Bansal Cygnus Hospital, Railway Road Karnal, Haryana, India
}

\begin{abstract}
Dengue infection may cause neurological complications ranging from encephalitis to radiculitis and result in disability and morbidity. We here present a case of Polyradiculomyelopathy with bilateral optic neuritis secondary to Dengue infection that showed minimal improvement despite timely steroids and plasma exchange.
\end{abstract}

Keywords: Dengue fever, Transverse myelitis, Steroids

\section{Introduction}

Dengue is a viral infection caused by Dengue virus which is an arbovirus of the Flavivirus genus and Flaviridae family. Dengue may present in any of its three forms-Classical Dengue fever, Dengue Hemorrhagic Fever and Dengue Shock Syndrome [1]. Until now, four serotypes have been isolated (DENV1-4). Polymerase chain reaction (PCR) technique is the method of choice for the detection of the viral genome. However, the ELISA method for detection of IgM antibodies for the dengue virus is the most widely used for epidemiological purposes [2]. NS1 Antigen has made the early diagnosis of Dengue possible as IgM may be negative in initial two to three days of the disease. Dengue fever is typically a self-limiting disease with a mortality rate of less than $1 \%$. When treated, dengue hemorrhagic fever has a mortality rate of $2-5 \%$. When left untreated, dengue hemorrhagic fever has a mortality rate as high as $50 \%$. Survivors usually recover without sequelae and develop immunity to the infecting serotype. In $20-30 \%$ of dengue hemorrhagic fever cases, the patient develops shock, known as the dengue shock syndrome.

\section{Case Report}

A 43 year old female, previously normotensive and nondiabetic, was brought to the Emergency Room of our hospital with complaints of fever of 10 days duration and weakness of all four limbs of one day duration. Patient had started with fever 10 days back associated with generalised aches and retro orbital pain. Patient had visited a local hospital where she was admitted in view of bleeding per vaginum and thrombocytopenia and diagnosed as a case of dengue fever. Patient had received platelet transfusions and other supportive treatment at the said hospital. Patient was discharged 7 days later in a stable condition. Two days later, while going to washroom at around 3 am in the morning, patient noticed weakness of her limbs. The weakness was profound as she had to drag herself to the bed. Patient also developed choking while drinking fluids and developed a low tone soft speech. On arrival to ER patient was drowsy but responded to verbal commands. Pupils on both sides were dilated and nonreactive to light. Vision was PL (perception to light) in both eyes. Fundus examination was normal. Bilateral gag reflexes were diminished. Motor examination revealed hypotonia in all muscles with generalized are flexia and diminished muscle power (MRCS II). Sensations could not be checked as patient was drowsy. Bilateral plantar responses were mute. NS1 antigen was positive. Other investigations are given in (Table 1). A diagnosis of Dengue Fever with polyradiculoneuropathy was made. Nerve conduction study was suggestive of a predominantly axonal, motor polyradiculoneuropathy affecting all four limbs. CSF analysis revealed 20 cells (all lymphocytes)

\begin{tabular}{|c|c|c|c|}
\hline Creatinine & $0.3 \mathrm{mg} / \mathrm{dl}$ & ANA & Negative \\
\hline HIV Serology & Negative & dsDNA & Negative \\
\hline CSF Analysis & & cANCA & Negative \\
\hline WBC & 20 cells/cumm & pANCA & Negative \\
\hline Differentials & Predominant Lymphos & dsDNA & Negative \\
\hline Glucose & $63.5 \mathrm{mg} / \mathrm{dl}$ & Anti NMO & Negative \\
\hline Protein & $99.83 \mathrm{mg} / \mathrm{dl}$ & & \\
\hline Gram Stain & Negative & & \\
\hline AFB Stain & Negative & & \\
\hline Culture & Negative & & \\
\hline Cryptococcus & Negative & & \\
\hline & Table 1: Investigations. & \\
\hline
\end{tabular}

with normal sugar and protein concentrations; Gram stain, AFB stain and cultures were negative. ADA was low. Contrast enhanced MRI spine showed a T2 hyperintensity in entire cervical cord extending cranially from medulla to D1 level distally (Figure 1). MRI brain showed multiple demyelinating lesions (Figure 2). A complete diagnosis of Myeloradiculoneuropathy with bilateral optic neuritis secondary to dengue fever was made. Motor functions within the hospital declined further with grade zero power in all four limbs and respiratory muscle involvement. Blood gas analysis revealed retention of $\mathrm{CO}_{2}$. Patient was electively intubated and put on ventilator support. In view of diffuse demyelination prognosis was kept guarded. Patient was started on high dose methylprednisolone pulse therapy (1 gram MP per day for 5 days). Patient however didn't show any response. In view of extensive disease she was started on plasma exchange. After a total of six exchanges patient showed a slight improvement in vision of right eye (perception of light to finger counting). Motor functions and vision in the left eye didn't improve. In view of persistent power deficits and respiratory muscle involvement, further treatment with intravenous immunoglobulin was planned. However family declined for IVIG. Presently patient persists with motor deficits (Grade 0 power all four limbs) and is on tracheostomy requiring intermittent ventilator support.

*Corresponding author: Waseem Dar, Department of Internal and Critical Care Medicine, Sanjeev Bansal Cygnus Hospital, Railway Road Karnal, Haryana, India, Tel: +918222800676; E-mail: drwaseem.mw@gmail.com

Received February 10, 2016; Accepted March 02, 2016; Published March 10 2016

Citation: Dar W, Sofi P, Ahmad R, Chauhan G, Singh S, et al. (2016) A Rare Complication of Dengue Fever. J Gen Pract 4: 237. doi: 10.4172/23299126.1000237

Copyright: @ 2016 Dar W, et al. This is an open-access article distributed under the terms of the Creative Commons Attribution License, which permits unrestricted use, distribution, and reproduction in any medium, provided the original author and source are credited. 


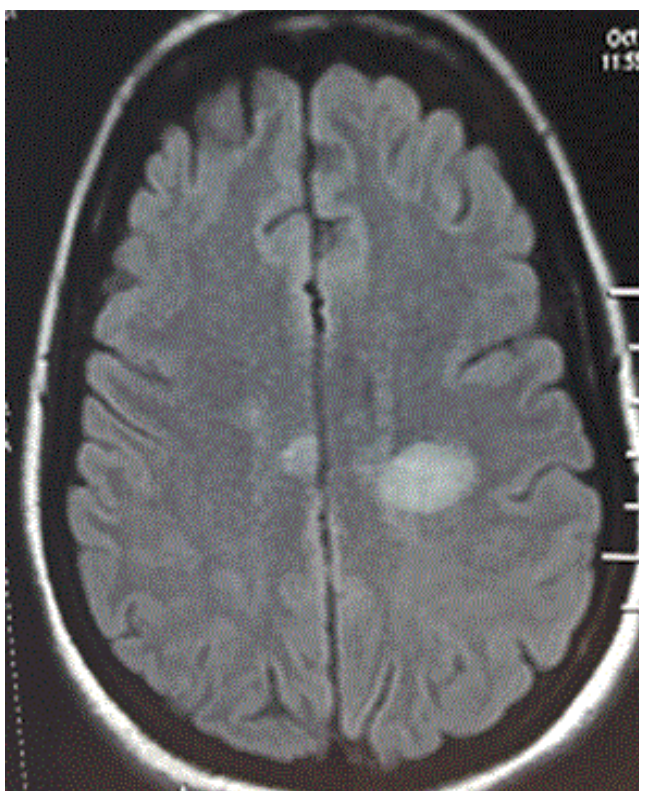

Figure 1: Showing hyper intense signals in brain in MRI brain.

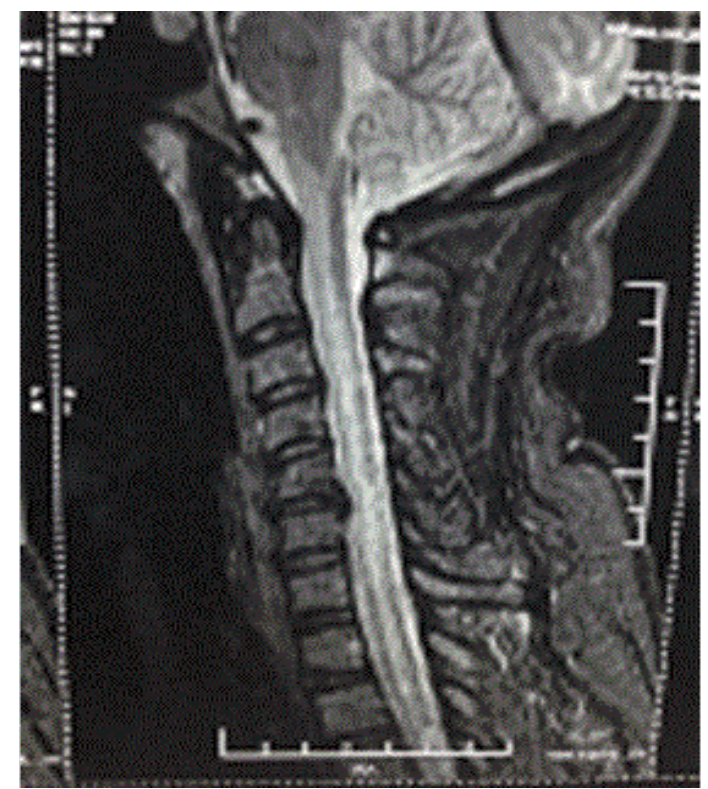

Figure 2: Showing hyper intense signals in cervical cord in MRI spine.

\section{Discussion}

Dengue fever may resolve without sequel or cause complications that increase morbidity and mortality. Neurological complications do occur in Dengue, albeit rarely [3]. They range from GBS to neuromyeloencephalitis to Opsoclonus-myoclonus syndrome [4]. The neuropathogenesis of DENV infection is still poorly understood. Viral and host factors may play an important role in the neurological disorders associated with Dengue. In this context, direct viral infection of central nervous system (CNS), autoimmune reaction, metabolic and hemorrhagic disturbances can be involved in the pathogenesis. Encephalitis is the most common neurological manifestation of dengue infection and the main symptoms include seizures, altered consciousness, and headaches.

Transverse myelitis occurring in Dengue fever is very rare and reported in a few case reports only [5]. Nouansompheng et al. reported a case of transverse myelitis in a 61 year old after 6 days of dengue fever. Treatment with intravenous pulse methylprednisolone and physiotherapy yielded a partial recovery, followed by complete resolution at 1 year post infection [6]. Similarly another case was reported by Manish et al. They reported a case of post dengue cervicothoracic myelitis that developed respiratory failure and succumbed to her illness despite steroid pulse therapy and other supportive treatment [7]. De Sousa et al. reported cluster of transverse myelitis following a dengue virus infection. From November 2004 to March 2005, 51 possible cases of neurological complications related to dengue were identified by the epidemiological surveillance of the State of Rondônia, Brazil. The diagnosis of acute transverse myelitis post-dengue was established in 26 patients, the majority were women, young and white. Antibodies against virus IgM were present in all cases and DEN 3 virus was isolated by PCR in one patient. Treatment with IV steroids was useful. Our patient however did not improve despite timely treatment.

\section{Conclusion}

Dengue infection is a common clinical problem in tropical countries particularly India given high prevalence of unhygienic conditions and poor sanitation facilities that result in a large number of breeding places and increased incidence of dengue. Neurological complications occur rarely in Dengue and may cause morbidity and sometimes mortality. Early treatment with steroids is important.

\section{References}

1. Bhatt S, Gething PW, Brady OJ, Messina JP, Farlow AW, et al. (2013) The global distribution and burden of dengue. Nature 496: 504-507.

2. Kassim FM, Izati MN, TgRogayah TA, Apandi YM, Saat Z (2011) Use of dengue NS1 antigen for early diagnosis of dengue virus infection. Southeast Asian J Trop Med Public Health 42: 562-569.

3. Verma R, Sharma P, Garg RK, Atam V, Singh MK, et al. (2011) Neurological complications of dengue fever: Experience from a tertiary center of north India. Annals of Indian Academy of Neurology 14: 272-278.

4. Puccioni-Sohler M, Orsini M, Soares CN (2012) Dengue: a new challenge for neurology. Neurol Int 4: 65-70.

5. Jackson ST, Mullings A, Bennett $F$ (2008) Dengue infection in patients presenting with neurological manifestations in a dengue endemic population West Indian Med J 57: 373-376.

6. Manish G, Nirdesh J, Sukriti K, Aniyang M (2013) A case of dengue fever complicated by acute transverse myelitis 6: 251-253.

7. De Sousa AM, Alvarenga MP, Alvarenga RMP (2014) A Cluster of Transverse Myelitis Following Dengue Virus Infection in the Brazilian Amazon Region. Tropical Medicine and Health 42: 115-120. 